\title{
Outcome of cases of Mitral Stenosis after Percutenuous Transvenous Mitral Commissurotomy (PTMC)
}

\section{Syed Ali Ahsan, ATM Iqbal Hasan, Sk.Marufuzzaman, M.A. Bari,}

This prospective study was carried out in the National Institute of Cardiovascular Diseases and Hospital (NIC VD), Dhaka, Bangladesh during the period January 2002 to December 2002. The result of percutenuous ballon mitral valvotomy were evaluated in 153 cases of mitral stenosis patients (mean age $36 \pm 15$ years) with symptomatic rheumatic mitral stenosis with single Inoue ballon technique PTMC results significant increase mitral valve area. 79.18 to $1.86 \pm .37$. Transmitral mean pressure gradient 19.6 \pm 8.9 to $3.7 \pm 38$, Decrease mean left atrial size $41.33 \pm 9.3$ to $30.56 \pm 8.16$ and Pulmonary artery systolic pressure $56 \pm 24$ to $39 \pm 17$, Peak pressure gradient (PPG) $19.6 \pm 8.9$ to 8.7 \pm 3.8 , But lower incidence of mitral regurgitation, only two cases developed MR grade III which were treated medically, one patient develop moderate haemopericardium which was treated conservatively. One patient was failed due to severe tight MS of valve area 0.5. No surgical intervention was needed.

It is concluded that percutenuous transvenous mitral commissurotomy (PTMC) is alternative to closed mitral commissurotomy (CMC) and intervention of choice for symptomatic rheumatic mitral stenosis with suitable mitral valve morphology.

Institute: National Institute of Cardiovascular Disease, Dhaka, Bangladesh, 